\title{
HPLC-UV Polyphenolic Profiles in the Classification of Olive Oils and Other Vegetable Oils via Principal Component Analysis
}

\author{
Mireia Farrés-Cebrián ${ }^{1}$, Raquel Seró ${ }^{1}$, Javier Saurina ${ }^{1,2}$ and Oscar Núñez 1,2,3,* \\ 1 Department of Chemical Engineering and Analytical Chemistry, University of Barcelona, \\ Martí i Franquès 1-11, E08028 Barcelona, Spain; mirefarres28@gmail.com (M.F.-C.); \\ rsero@ub.edu (R.S.); xavi.saurina@ub.edu (J.S.) \\ 2 Research Institute in Food Nutrition and Food Safety, University of Barcelona, Recinte Torribera, \\ Av. Prat de la Riba 171, Edifici de Recerca (Gaudí), E08921 Santa Coloma de Gramenet, Barcelona, Spain \\ 3 Serra Húnter Program, Generalitat de Catalunya, Rambla de Catalunya 19-21, E08007 Barcelona, Spain \\ * Correspondence: oscar.nunez@ub.edu; Tel.: +34-93-403-3706
}

Academic Editor: Didier Thiébaut

Received: 6 October 2016; Accepted: 1 December 2016; Published: 8 December 2016

\begin{abstract}
High performance liquid chromatography-ultraviolet (HPLC-UV) was applied to the analysis and characterization of olive oils and other vegetable oils. A chromatographic separation on a Zorbax Eclipse XDB-C8 reversed-phase column was proposed under gradient elution, employing $0.1 \%$ formic acid aqueous solution and methanol as mobile phase, for the determination of 14 polyphenols and phenolic acids, allowing us to obtain compositional profiles in less than 20 min. Acceptable sensitivity (limit of detection (LOD) values down to $80 \mu \mathrm{g} / \mathrm{L}$ in the best of cases), linearity ( $r^{2}$ higher than 0.986 ), good run-to-run and day-to-day precisions (relative standard deviation (RSD) values lower than $11.5 \%$ ), and method trueness (relative errors lower than $6.8 \%$ ) were obtained. The proposed HPLC-UV method was then applied to the analysis of 72 oil samples (47 olive oils and 27 vegetable oils including sunflower, soy, corn, and mixtures of them). Analytes were recovered using a liquid-liquid extraction method employing ethanol:water 70:30 $(v / v)$ solution and hexane as extracting and defatting solvents, respectively. HPLC-UV polyphenolic profiles using peak areas were then analysed by principal component analysis (PCA) to extract information from the most significant data contributing to the characterization and classification of olive oils against other vegetable oils, as well as among Arbequina and Picual olive oil varieties. PCA results showed a noticeable difference between olive oils and the other classes. In addition, a reasonable discrimination of olive oils as a function of fruit varieties was also encountered.
\end{abstract}

Keywords: high performance liquid chromatography; UV-detection; polyphenols; principal component analysis; food characterization; olive oils; vegetable oils

\section{Introduction}

The health of the individual and the population in general is the result of interactions between genetics and a number of environmental factors, nutrition being one of the factors of major importance [1,2]. An appropriate diet can prevent non-communicable disease linked to chronic inflammation such as cancer, diabetes, metabolic syndrome, obesity, pulmonary diseases, and cognition disorders. The importance of incorporating functional foods in the diet or following beneficial dietary patterns to improve health is fairly well-demonstrated [3]. Dietary patterns comprise all foods and beverages consumed in various characteristic combinations by different populations across the world. Among the dietary patterns that are known to have beneficial health effects, the Mediterranean diet is the best-studied and most evidence-based diet for preventing not only cardiovascular diseases but 
also other chronic diseases, and today has become a standard for healthy eating and a dietary template of particular value [4]. Moreover, due to its widely proven beneficial health effects, the Mediterranean diet has been on UNESCO's Representative List of the Intangible Cultural Heritages of Humanity since 2013 [5].

Olive oil has been produced for about 6000 years, but in the last 30 years there has been a growing interest in the use of olive oil in cooking due to the increased knowledge about Mediterranean food and public awareness of the health benefits of the Mediterranean diet and, particularly, of olive oil [6]. In fact, virgin olive oil (VOO) is the primary fat source and a fundamental component of the Mediterranean diet. VOO is obtained through physical procedures either by mechanical or by direct pressing of the pulp of the olive fruit (Olea europaea L.). The olives, after being crushed to form a pomace, are homogenized and pressed. This oil is not subjected to any other treatment than washing, decantation, centrifugation or filtration [7]. The oil produced from this first press is known as extra-virgin olive oil (EVOO), and it is the highest qualitative olive oil containing the highest levels of beneficial constituents [8]. Apart from the natural unrefined state, olive oil can also be consumed as a refined product which is made either from virgin olive oil and called refined olive oil (ROO) or refined olive-pomace oil [7], or from solvent-extracted oil [9] which is called refined husk oil (RHO).

Regarding EVOO constituents, triglycerides are the major components, representing more than $98 \%$ of the total weight. The fatty acid composition of triglycerides is $11 \%$ saturated fatty acids (e.g., palmitic acid (C16:0)), around 80\% monounsaturated fatty acids (being oleic acid (C18:1), and from $55 \%$ to $83 \%$ total fatty acids, the most important feature of olive oils in comparison to other vegetable oils), and $9 \%$ polyunsaturated fatty acids (i.e., linoleic acid (C18:2 $\omega 6))$ [10,11]. In addition to the fatty acids, EVOO contains antioxidant and anti-inflammatory compounds, such as squalene, phytosterols, and mainly polyphenols and phenolic acids, which provide important beneficial health effects $[12,13]$. The total amount of polyphenols and phenolic acids in olive oils depends on several parameters, such as the growing area, cultivation techniques, water resources, soil management, degree of maturation, and climate, but the oil production method (malaxation and extraction) also affects the final amount of these compounds [14-16]. Apart from the sensory and functional properties attributed to these compounds, their impact on the characterization, classification, and authentication studies cannot be underestimated [17]. Polyphenol and phenolic acid contents seem to be related to several food features such as the cultivar geographical areas, variety, and extraction conditions, and as a result, polyphenol content can be exploited as a source of analytical data to establish vegetable oil product classifications and even to achieve detection of product adulterations.

Different analytical methodologies have been developed for the determination of polyphenols and phenolic acids in vegetable oils $[18,19]$. In this kind of matrix, one of the most critical points is the extraction procedure employed because it will influence the results obtained after analysis. Liquid-liquid extraction (LLE) and solid phase extraction (SPE) are among the most widely used extraction methods in the determination of polyphenols in olive oils [18-28]. As regards the analytical technique, chromatographic and electrophoretic methods are employed to achieve separation and quantification of these compounds after sample extraction. However, the fact that a derivatization step is commonly necessary when gas chromatography (GC) is employed [21], and the low sensitivity attainable with capillary electrophoresis (CE) methods $[29,30]$ in the determination of polyphenols make liquid chromatography (LC) the most widely employed technique for that purpose either with UV detection [22,31,32] or coupled to mass spectrometry (LC-MS) systems [20,33-35]. Liquid chromatography in combination with high-resolution mass spectrometry (HRMS) has also been described for the identification and determination of phenolic compounds in vegetable oils $[23,24,28,36]$. Despite the number of applications described in the literature dealing with the determination of polyphenols in vegetable oil samples by LC, few works are devoted to classification and authentication purposes [25].

The aim of this work is to develop an economic, simple, and robust high-performance liquid chromatography (HPLC) method with UV detection for the determination of several polyphenols and 
phenolic acids in a variety of vegetable oils, including olive, sunflower, soy, and corn oils as well as vegetable oils prepared by mixtures of different seeds and fruits. HPLC-UV polyphenolic profiles using peak area data will be exploited to carry out exploratory principal component analysis (PCA) to achieve vegetable oil sample characterization and classification.

\section{Materials and Methods}

\subsection{Chemicals and Standards}

Unless otherwise specified, analytical grade reagents were used. The polyphenols and phenolic acids studied, which are shown in Table 1, were all obtained from Sigma-Aldrich (Steinheim, Germany) except for rosmanol, which was purchased from Cymit Quimica S.L. (Barcelona, Spain). Stock standard solutions of all polyphenols and phenolic acids at ca. $1000 \mathrm{mg} / \mathrm{L}$ were prepared in methanol and store in amber glass vials. The intermediate working solutions were prepared weekly by dilution of these stock standard solutions using Milli-Q water. All stock solutions were stored at $4{ }^{\circ} \mathrm{C}$ for no more than 1 month.

Methanol (Chromasolv ${ }^{\circledR}$ for HPLC, $\geq 99.9 \%$ ), hexane, and formic acid ( $\left.\geq 98 \%\right)$ was also obtained from Sigma-Aldrich, and ethanol (absolute) was purchased from VWR International Eurolab S.L. (Barcelona, Spain).

The water used in this work was purified by employing an Elix 3 coupled to a Milli-Q purification system (Millipore, Bedford, MA, USA) and filtered through a $0.22 \mu \mathrm{m}$ nylon membrane integrated into the Milli-Q system.

Table 1. Chemical structures of studied polyphenols and phenolic acids.

\begin{tabular}{|c|c|c|c|c|}
\hline Peak & Compound & Family & Structure & CAS Number \\
\hline 1 & Homogentisic acid & Phenolic acid & & $451-13-8$ \\
\hline 2 & (+)-Catechin hydrate & Flavanol & & $225937-10-0$ \\
\hline 3 & Tyrosol & Phenolic alcohol & & 501-91-0 \\
\hline 4 & 4-Hydroxybenzoic acid & Phenolic acid & & 99-96-7 \\
\hline 5 & Caffeic acid & Phenolic acid & & $331-39-5$ \\
\hline 6 & Syringic acid & Phenolic acid & & $530-57-4$ \\
\hline 7 & Vanillin & Phenolic aldehyde & & $121-33-5$ \\
\hline
\end{tabular}


Table 1. Cont.

Peak Compound
Syringaldehyde
Sinapic acid
Ferulic acid
10
Veratric acid

\subsection{Instrumentation and Methods}

Chromatographic separation was performed on a HPLC instrument from Dionex (Dionex Softron $\mathrm{GmbH}$, Gemering, Germany) equipped with a P680 pump, an ASI-100 automatic sample injector, a UVD170U UV-Vis detector, and a TCC-100 thermostatted column compartment. A Zorbax Eclipse XDB-C8 reversed-phase column $(150 \times 4.6 \mathrm{~mm}$ i.d., $5 \mu \mathrm{m}$ particle size $)$ provided by Agilent Technologies (Santa Clara, CA, USA) was used for the proposed method. A $0.1 \%(v / v)$ formic acid aqueous solution (solvent $\mathrm{A}$ ) and methanol (solvent $\mathrm{B}$ ) were used to establish the gradient elution as follows: 0-2 min at 10\% B (initial conditions); $2-4.5$ min linear gradient from $10 \%$ B to $25 \% \mathrm{~B} ; 4.5-7 \mathrm{~min}$ at $25 \% \mathrm{~B} ; 7-22 \mathrm{~min}$ linear gradient from $25 \% \mathrm{~B}$ to $90 \% \mathrm{~B} ; 22-24 \mathrm{~min}$ at $90 \% \mathrm{~B} ; 24-25 \mathrm{~min}$ back to the initial conditions at $10 \% \mathrm{~B}$; and $25-30 \mathrm{~min}$ at $10 \% \mathrm{~B}$ for column equilibration. The mobile phase flow rate was $1 \mathrm{~mL} \cdot \mathrm{min}^{-1}$ and the injection volume was $10 \mu \mathrm{L}$. Direct $\mathrm{UV}$ absorption detection was carried out at $257 \mathrm{~nm}$ (for 4-hydroxybenzoic and veratric acids, and kaempferol), $280 \mathrm{~nm}$ (for catechin, tyrosol, vanillin, rosmanol, and homogentisic and syringic acids) and $316 \mathrm{~nm}$ (for syringaldehyde and caffeic, p-coumaric, sinapic, and ferulic acids). Chromeleon software version 6.7 (Dionex, Softron GmbH, Gemering, Germany) was used for HPLC system control and data acquisition.

\subsection{Samples and Sample Treatment}

The set of samples to be analysed consisted of the following vegetable oils from different trademarks purchased from Barcelona markets: 47 olive oils, 16 sunflower oils, 2 corn oils, 2 soy oils, and 5 vegetable oils produced from mixtures of seeds (3 sunflower/corn oils and 2 sunflower/soy oils). Among the 47 analysed olive oils, 10 were obtained from Arbequina olives and 5 from Picual 
olives. No information regarding the olive variety was provided by producers regarding the other 32 olive oils.

Sample treatment was carried out following a previously described method with some modifications [20]. Briefly, $2.00 \mathrm{~g}$ of vegetable oil were weighed into a $15 \mathrm{~mL}$ polytetrafluoroethylene (PTFE) centrifuge tube (Serviquimia, Barcelona, Spain), and $2 \mathrm{~mL}$ of an ethanol:water 70:30 $v / v$ solution were added. The mixture was shaken vigorously for 2 min using a vortex mixer (Stuart, Stone, UK). The extract was then centrifuged at $3500 \mathrm{rpm}$ for $5 \mathrm{~min}$ using a Rotanta $460 \mathrm{RS}$ centrifuge (Hettich, Tuttlingen, Germany). Then the mixture was frozen for $24 \mathrm{~h}$ at $-18{ }^{\circ} \mathrm{C}$ to facilitate the quantitative recovery of the extract solution. The corresponding extract was transferred into another $15 \mathrm{~mL}$ PTFE centrifuge tube for further clean-up. Then, $2 \mathrm{~mL}$ of hexane were added as the defatting agent and the mixture was shaken vigorously for $2 \mathrm{~min}$ in a vortex mixer and centrifuged at $3500 \mathrm{rpm}$ for $5 \mathrm{~min}$. Then, the aqueous ethanolic extracts were directly analysed with the proposed HPLC-UV method.

Also, a quality control (QC) consisting of a mixture of $50 \mu \mathrm{L}$ of each ethanolic aqueous sample extract was prepared to evaluate the repeatability of the method and the robustness of the chemometric results.

\subsection{Data Analysis}

SOLO chemometric software from Eigenvector Research was used for calculations with principal component analysis (PCA) [37]. A detailed description of the theoretical background of this method is given elsewhere [38].

Data matrices to be treated consisted on the peak area values of the 14 studied polyphenols and phenolic acids detected (at the registered wavelengths) in the different samples under study. Thus, the dimension of the matrix was 72 samples $\times 14$ analytes. Normalization pretreatment with respect to the overall polyphenolic concentration was applied to provide similar weighs to all the samples. For additional details see Reference [39].

Peak identification in the analysed vegetable oil samples was performed by comparison of retention times and UV spectra with those of a polyphenolic standard solution. Peak purity was confirmed by comparison of UV spectra through the entire peak signal.

\section{Results and Discussion}

\subsection{HPLC Conditions}

The present work aimed to develop a HPLC-UV method for the separation and determination of fourteen polyphenols and phenolic acids. We first employed a gradient elution program with an increasing methanol component from 10\% to $90 \%$ over $19 \mathrm{~min}$ (after an isocratic step of $1 \mathrm{~min}$ at initial conditions), followed by an isocratic step of $3 \mathrm{~min}$ at $90 \%$. The HPLC-UV chromatogram obtained under these conditions for a standard mixture of $\sim 25 \mathrm{mg} / \mathrm{L}$ of studied compounds in water as well as the gradient profile are shown in Figure 1a. The gradient elution program continued up to $30 \mathrm{~min}$ to get back to mobile phase initial conditions and to guarantee column re-equilibration. As can be seen, baseline chromatographic separation was achieved for almost all compounds in less than $17 \mathrm{~min}$, although coelutions between vanillin and syringaldehyde (peaks 7 and 8, respectively) and between $p$-coumaric, sinapic and ferulic acids (peaks 9,10 and 11, respectively) occurred.

The gradient elution program was modified in order to improve the chromatographic separation of the fourteen studied compounds. For that purpose, methanol content was increased more slowly from $10 \%$ to $25 \%$ (from $\min 1$ to $\min 7$ ), and then up to $90 \%$ methanol (from $\min 7$ to $\min 20$ ). The chromatographic separation obtained is shown in Figure 1b. From this modification, a full resolution of vanillin and syringaldehyde was attained and a considerable improvement on the separation between $p$-coumaric and sinapic acids (peaks 9 and 10, respectively) was achieved, although partial coelution of these two last phenolic acids with ferulic acid still persisted. Regarding analysis time, a small increase up to 19 min was obtained. 
Up to this point, several combinations of linear gradients and isocratic steps were evaluated to try to get a full resolution of all compounds. It should be mentioned that none of the gradient programs evaluated led to the baseline separation of $p$-coumaric, sinapic, and ferulic acids, even when increasing analysis time. In all the conditions studied, sinapic acid coeluted either with $p$-coumaric acid or with ferulic acid. For this reason, as a compromise between analysis time and peak chromatographic resolution, the gradient elution program finally selected consisted of an isocratic step at initial conditions ( $10 \%$ methanol) for $2 \mathrm{~min}$, a linear gradient up to $25 \%$ methanol in $2.5 \mathrm{~min}$, an isocratic step at $25 \%$ methanol for $2.5 \mathrm{~min}$, and then up to $90 \%$ methanol till $22 \mathrm{~min}$.

Figure 1c shows the HPLC-UV chromatogram obtained under these conditions. Although partial coelutions are still observed between $p$-coumaric, sinapic and ferulic acids, the chromatographic separation was considered acceptable for the intended purposes.
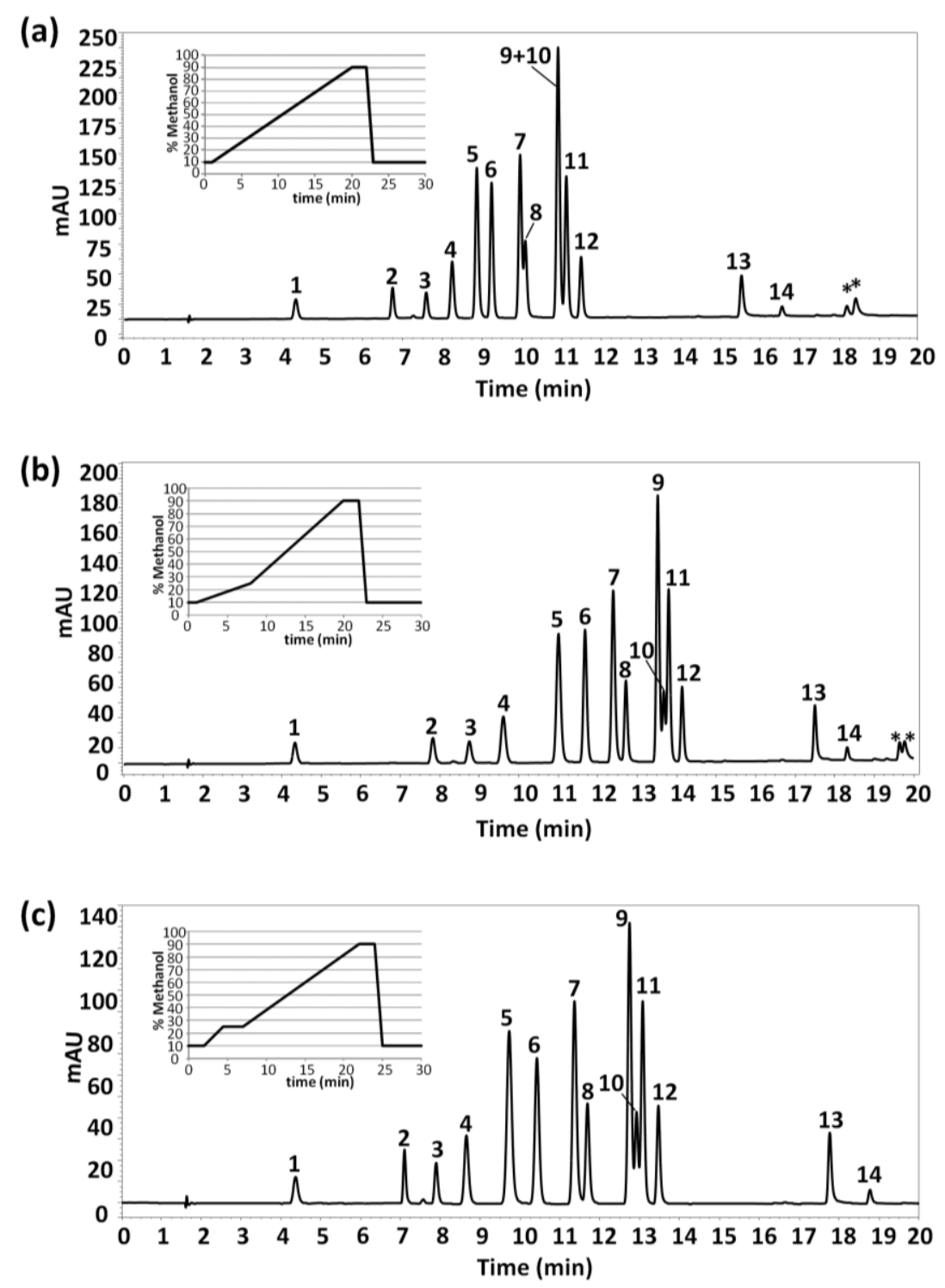

Figure 1. High performance liquid chromatography-ultraviolet (HPLC-UV) chromatograms of a mixture of studied polyphenols ( $25 \mathrm{mg} / \mathrm{L}$ in water) under different gradient elution conditions. UV detection: $\lambda 280 \mathrm{~nm}$. Peak identification: 1, homogentisic acid; 2, (+)-catechin hydrate; 3, tyrosol; 4, 4-hydroxybenzoic acid; 5, caffeic acid; 6, syringic acid; 7, vanillin; 8, syringaldehyde; 9, $p$-coumaric acid; 10, sinapic acid; 11, ferulic acid; 12, veratric acid; 13, kaempferol; and 14, rosmanol. $\left.{ }^{*}\right)$ system peaks. 


\subsection{Instrumental Quality Parameters}

The proposed HPLC-UV method was validated by determining instrumental quality parameters under optimal conditions. Figures of merit are summarized in Table 2. The LODs, based on a signal-to-noise ratio of 3:1, were obtained by analyzing polyphenol standard solutions at decreasing concentration levels. Most of the analysed polyphenols showed LOD values below $0.9 \mathrm{mg} / \mathrm{L}$ (in the range between $80 \mu \mathrm{g} / \mathrm{L}$ and $900 \mu \mathrm{g} / \mathrm{L}$ ), and only five polyphenols showed lower sensitivity, being rosmanol the one with the poorest LOD $(11.5 \mathrm{mg} / \mathrm{L})$. The limits of quantification (LOQs) determined, based on a signal-to-noise ratio of 10:1, were in the range of 0.25 to $38.4 \mathrm{mg} / \mathrm{L}$.

Run-to-run and day-to-day precisions for polyphenol quantification were calculated at a concentration level of $\sim 30 \mathrm{mg} / \mathrm{L}$. In order to estimate the run-to-run precision, five replicate determinations were carried out using the proposed method under optimal conditions. The relative standard deviations ( $\%$ RSDs) were between $0.6 \%$ and $2.9 \%$.

Table 2. HPLC-UV instrumental quality parameters.

\begin{tabular}{|c|c|c|c|c|c|c|c|c|c|c|}
\hline \multirow[b]{2}{*}{ Compound } & \multirow{2}{*}{$\begin{array}{l}\text { LOD } \\
(\mathrm{mg} / \mathrm{L})\end{array}$} & \multirow{2}{*}{$\begin{array}{c}\text { LOQ } \\
(\mathrm{mg} / \mathrm{L})\end{array}$} & \multirow{2}{*}{$\begin{array}{l}\text { Linearity } \\
\qquad\left(r^{2}\right)\end{array}$} & \multicolumn{2}{|c|}{$\begin{array}{l}\text { Run-to-Run Precision } \\
\quad(\text { RSD } \%, n=5)\end{array}$} & \multicolumn{2}{|c|}{$\begin{array}{l}\text { Day-to-Day Precision } \\
(\text { RSD } \%, n=5 \times 3)\end{array}$} & \multicolumn{3}{|c|}{ Trueness $(n=5)$} \\
\hline & & & & $t_{\mathrm{r}}$ & Concentration & $t_{\mathrm{r}}$ & Concentration & $\begin{array}{l}\text { Conc. } \\
\text { value } \\
(\mathrm{mg} / \mathrm{L})\end{array}$ & $\begin{array}{c}\text { Calc. } \\
\text { value } \\
(\mathrm{mg} / \mathrm{L})\end{array}$ & $\begin{array}{c}\text { Error } \\
(\%)\end{array}$ \\
\hline 1) Homogentisic acid & 2.6 & 8.7 & 0.987 & 0.12 & 2.9 & 0.32 & 4.3 & 4.3 & 29.3 & 4.5 \\
\hline 2) (+)-catechin hydrate & 3.5 & 11.6 & 0.995 & 0.05 & 1.1 & 0.16 & 10.1 & 10.1 & 31.9 & 2.3 \\
\hline 3) Tyrosol & 3.3 & 11.0 & 0.986 & 0.05 & 2.0 & 0.15 & 7.3 & 7.3 & 35.1 & 6.8 \\
\hline 5) Caffeic acid & 0.5 & 1.5 & 0.992 & 0.07 & 0.6 & 0.23 & 4.2 & 4.2 & 41.0 & 6.7 \\
\hline 6) Syringic acid & 0.5 & 1.7 & 0.996 & 0.05 & 0.7 & 0.20 & 6.6 & 6.6 & 33.2 & 3.9 \\
\hline 7) Vanillin & 0.6 & 1.9 & 0.995 & 0.04 & 0.6 & 0.14 & 7.7 & 7.7 & 31.1 & 2.8 \\
\hline 8) Syringaldehyd & 0.9 & 3.2 & 0.988 & 0.04 & 1.0 & 0.13 & 4.8 & 4.8 & 33.4 & 2.8 \\
\hline 9) $p$-coumaric acid & 0.08 & 0.25 & 0.997 & 0.04 & 0.8 & 0.12 & 3.0 & 3.0 & 33.4 & 0.9 \\
\hline 10) Sinapic acid & 0.15 & 0.5 & 0.995 & 0.04 & 0.6 & 0.11 & 5.7 & 5.7 & 33.1 & 2.4 \\
\hline 11) Ferulic acid & 0.08 & 0.27 & 0.999 & 0.04 & 0.6 & 0.11 & 11.5 & 11.5 & 32.6 & 3.2 \\
\hline
\end{tabular}

${ }^{\text {a }}$ Calculated at a concentration $\sim 30 \mathrm{mg} / \mathrm{L}$.

A total of 15 replicate determinations were carried out on three non-consecutive days to calculate the day-to-day precision. As expected, RSD values slightly higher than those observed for run-to-run precision were achieved, with percentages between $3.0 \%$ and $11.5 \%$. Run-to-run and day-to-day precisions of retention times were also calculated obtaining RSD values lower than $0.12 \%$ and $0.32 \%$, respectively, thus showing the good precision performance of the proposed HPLC-UV method.

External calibration curves based on peak areas at concentrations between LOQ and $80 \mathrm{mg} / \mathrm{L}$ were built. Good linearity was observed $\left(r^{2}>0.986\right)$ for all the compounds. The trueness of the proposed HPLC-UV method was also evaluated via quintuplicate analyses, using external calibration, of standard solutions at concentrations of $\sim 30 \mathrm{mg} / \mathrm{L}$, achieving very good results, with relative absolute errors ranging from $0.3 \%$ to $8.3 \%$.

\subsection{Exploratory Analysis by PCA}

PCA provided plots of scores and loadings, showing the distribution of the samples and variables employed on the principal components (PCs), respectively. The study of the plot of scores revealed patterns that may be correlated to sample characteristics, such as the type of seed employed on the vegetable oil, etc. The study of the distribution of variables from the plot of loadings provided information dealing with their correlations as well as dependencies of polyphenols and phenolic acids on vegetable oil.

Characterization of vegetable oils was attempted using the peak areas of the fourteen polyphenols and phenolic acids under study found in the analysed samples. For that purpose, the 72 vegetable 
oil samples were processed as indicated in the sample treatment section and the resulting extracts were randomly analysed with the proposed HPLC-UV method. Figure 2 shows the HPLC-UV chromatograms of (a) an extra virgin olive oil sample within the 47 olive oils analysed; (b) a sunflower oil sample; (c) a soybean oil sample; and (d) a corn oil sample, registered at $280 \mathrm{~nm}$ under optimal conditions.
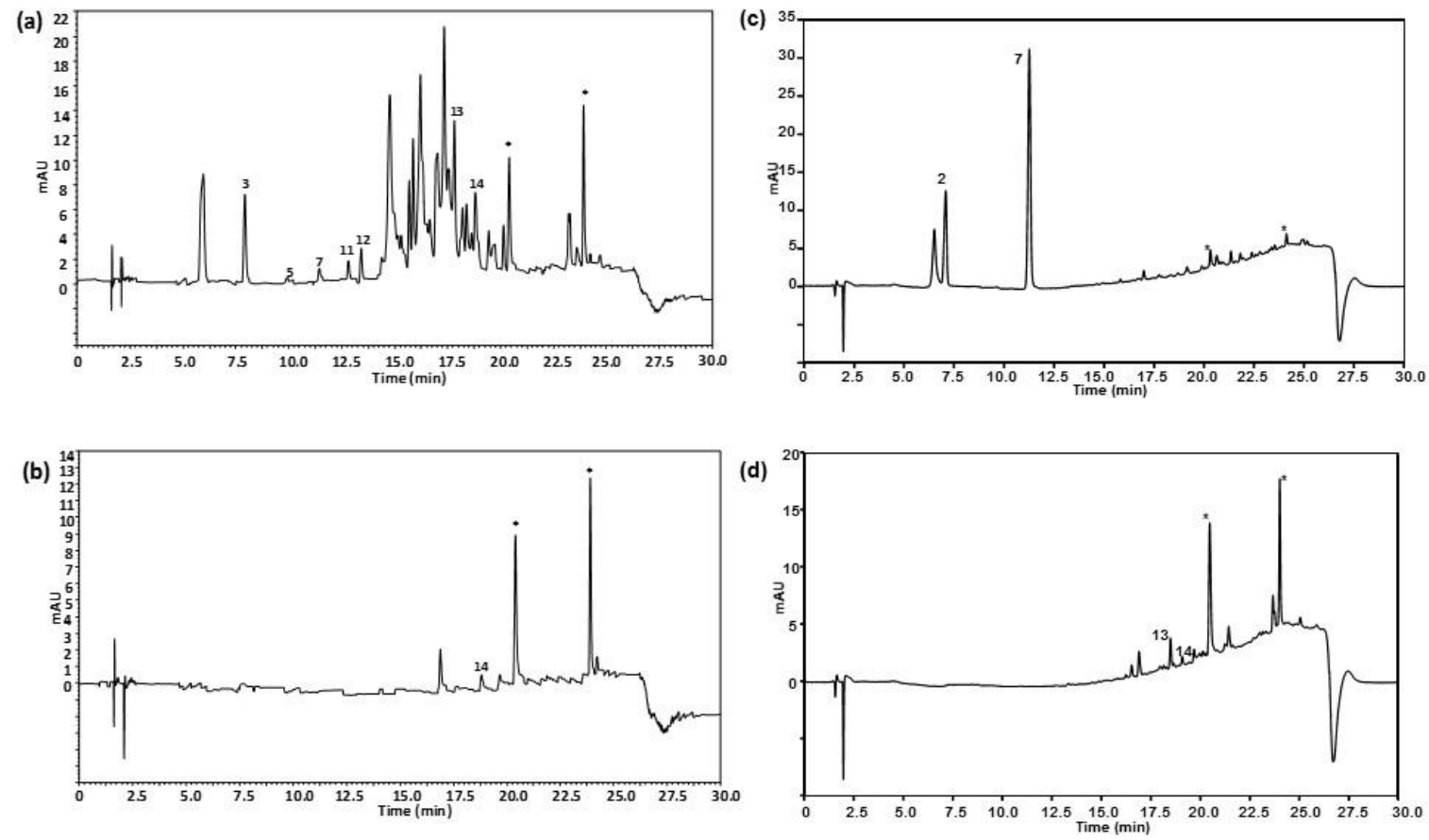

Figure 2. HPLC-UV chromatograms of (a) an extra virgin olive oil sample within the 47 olive oils analysed (olive type not specified); (b) a sunflower oil sample; (c) a soy oil sample, and (d) a corn oil sample, obtained under optimal conditions. UV detection: $\lambda 280 \mathrm{~nm}$. Peak identification as in Figure 1.

As can be seen, chromatographic profiles are very different, olive oils being richer in absorbing signals in comparison to the other vegetable oils. Additionally, only a few of the fourteen analysed polyphenols were detected being tyrosol, caffeic acid, vanillin, ferulic acid, veratric acid and kaempferol, and in a lower proportion rosmanol, the most frequently found in olive oils. This is the first time that veratric acid, kaempferol and rosmanol have been detected in extra virgin olive oils. This is only a tentative identification of these chemical compounds and further investigations will be carried out to confirm their presence. Regarding other oils, rosmanol, and in some cases vanillin among others, prevailed in sunflower and the other seed-based oils. With the information of the detected targeted polyphenols and phenolic acids, a peak area data matrix with a dimension of 72 samples $\times 14$ compounds was built to be treated by PCA. Figure 3 shows the scatter plots of scores and loadings of PC1 vs. PC2 of the model so obtained.

As can be seen, QCs appeared in a compact group in the center of the graph, thus demonstrating the good repeatability and robustness of the proposed method. Regarding this preliminary exploratory classification model, vegetable oils other than olive oils appeared in a very compact group in the center-left area of the PC1 vs. PC2 plot, and from this point olive oils were widely distributed to the right of the plot, showing that PCA employing HPLC-UV polyphenolic profiles targeting only 14 simple polyphenols and phenolic acids allows discrimination between olive oils and other vegetable oils.

The attention was then focused only on olive oil samples to see if some discrimination occurred regarding the type variety of olives. Figure 4 shows, as an example, the HPLC-UV chromatograms registered at $280 \mathrm{~nm}$ under optimal conditions of (a) an EVOO obtained from Arbequina olives and (b) an EVOO obtained from Picual olives. As can be seen, the chromatographic profiles were quite 
different, with Picual olive oils characterized to present more intense peak signals between 14 and 20 min in comparison to Arbequina olive oils.
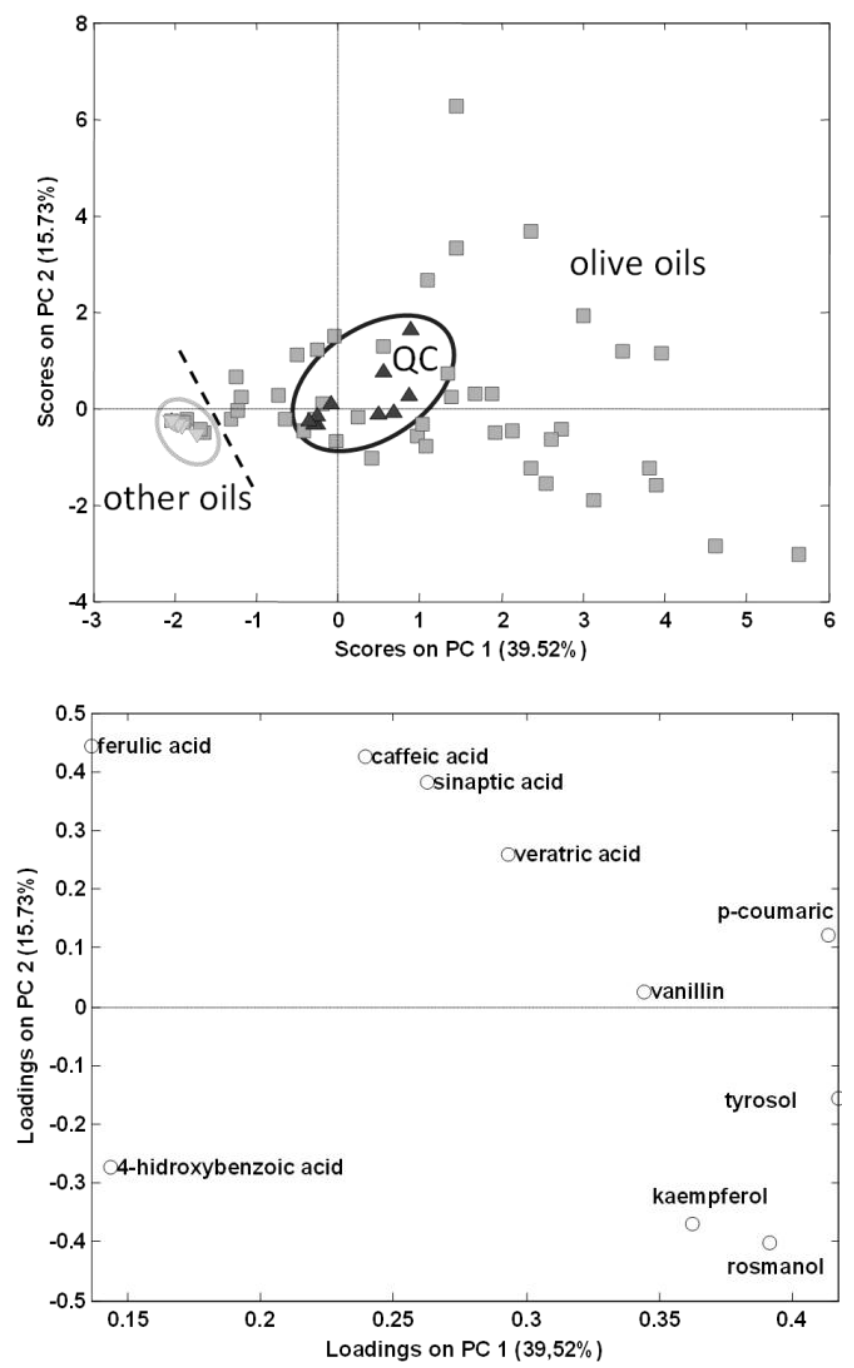

Figure 3. Principal component analysis (PCA) results (score and loading plots of PC1 vs. PC2) using as analytical data the normalized peak areas of the fourteen targeted polyphenols and phenolic acids found in the analysed vegetable oils. $\square$ olive oils, $\nabla$ other vegetable oils, and $\boldsymbol{\Delta}$ quality controls.

A PCA model was then made only using EVOOs; to do this, peak areas of targeted polyphenols and phenolic acids in the olive oil samples were used to build a data matrix with a dimension of 47 samples $\times 14$ compounds to be subjected to PCA. The scatter plots of scores and loadings of PC1 vs. PC2 are shown in Figure 5. PC1 is discriminating samples according to the content on analysed polyphenols, being samples distributed to the right area of the plot richer in the targeted compounds than samples located to the left. In contrast, it seems that PC2 is differentiating samples according to the variety of olive employed, with 100\% Arbequina olive oils located to the top area of the plot, while $100 \%$ Picual olive oils tend to be located to the bottom area. Information regarding type of olive fruits employed in the production of the other olive oil samples analysed was not provided by the producers, and most of them will probably be obtained from mixtures of different varieties. 

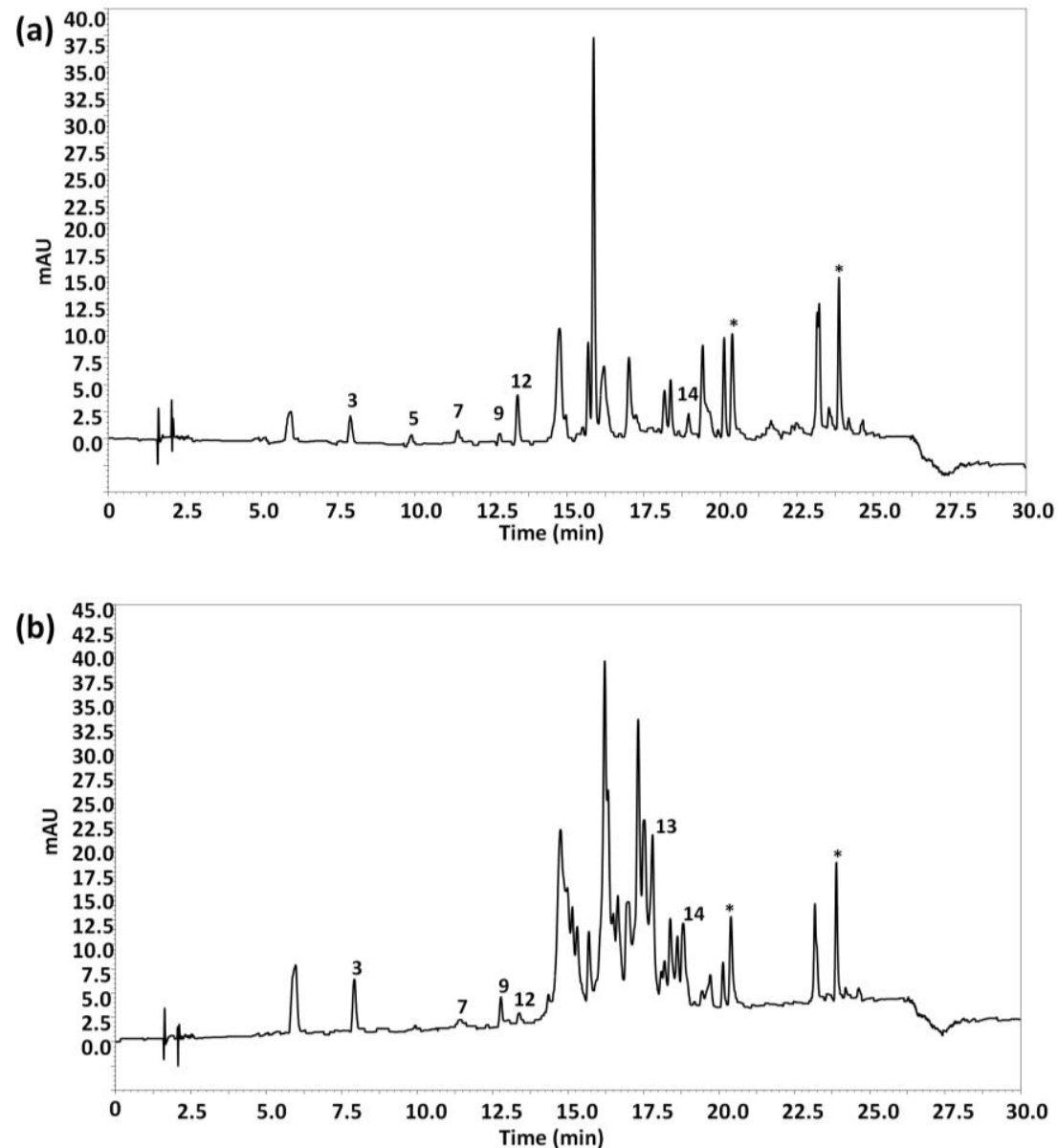

Figure 4. HPLC-UV chromatograms of (a) an extra-virgin olive oil (EVOO) obtained from $100 \%$ Arbequina olives and (b) an EVOO obtained from 100\% Picual olives, obtained under optimal conditions. UV detection: $\lambda 280 \mathrm{~nm}$. Peak identification as in Figure 1.

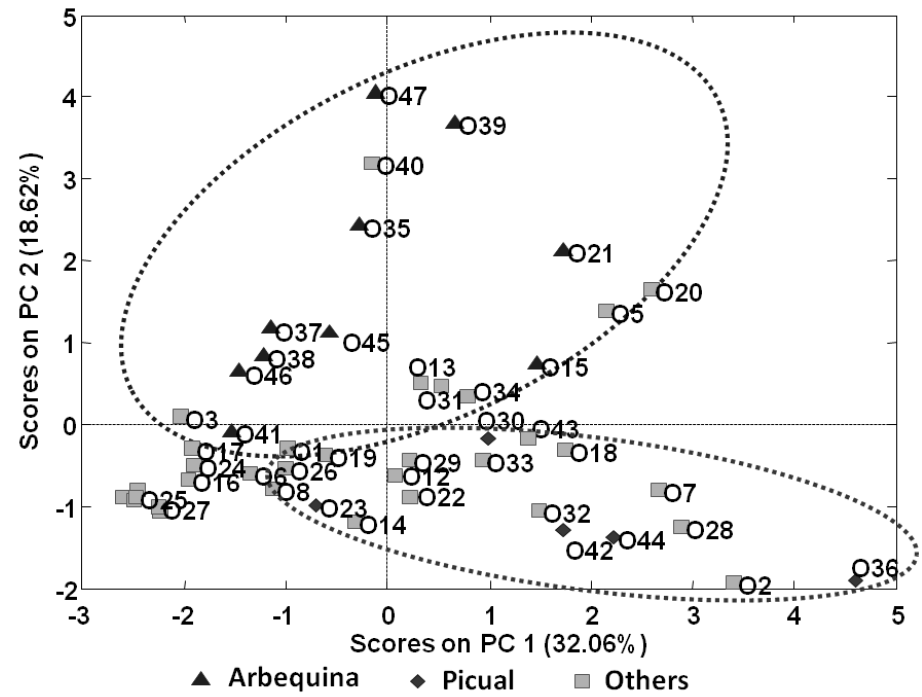

Figure 5. Cont. 


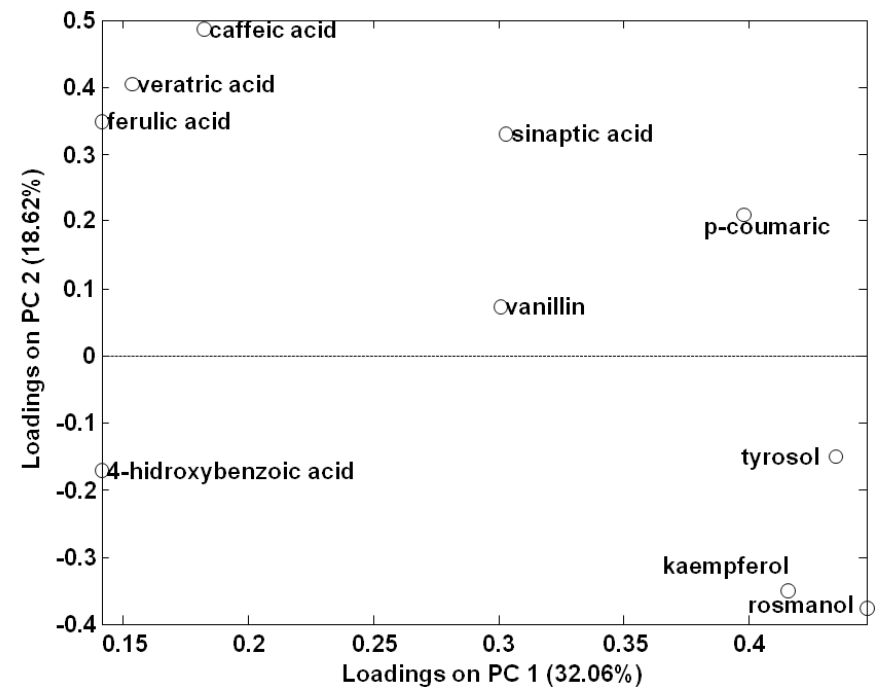

Figure 5. PCA results (score and loading plots of PC1 vs. PC2) using as analytical data the normalized peak areas of the fourteen targeted polyphenols and phenolic acids found in the analysed olive oils.

\section{Conclusions}

The results obtained in this work show that HPLC-UV polyphenolic profiles can be proposed as the analytical data for a simple and suitable characterization and classification of olive oils and other vegetable oils. HPLC-UV method performance was evaluated, producing acceptable LOD and LOQ values, with good linearity, run-to-run and day-to-day precisions, and method trueness.

PCA applied to phenolic peak area data and the resulting models provided an acceptable classification and discrimination between olive oils with respect to any other kind of vegetable oil sample, although no differentiation among these latter was achieved. In addition, an acceptable discrimination among olive oils regarding the olive variety was also observed, being possible also to slightly classify samples among Arbequina and Picual olives.

Exploratory PCA of the data obtained by HPLC-UV demonstrated that the method was useful for the discrimination of olive oil and other vegetable oils, and it could be employed in future studies to identify olive oil samples adulterated with other vegetable oils of poorer quality, as well as a starting point to guarantee the Protected Designation of Origin of these products. Future experiments will be carried out in this sense by employing partial least square regression to resolve authentication and adulteration issues.

Acknowledgments: The authors gratefully acknowledge the financial support received from the Spanish Ministry of Economy and Competitiveness under the projects CTQ2014-65324 and CTQ2015-63968-C2-1-P, and from the Agency for Administration of University and Research Grants (Generalitat de Catalunya, Spain) under the projects 2014 SGR-377 and 2014 SGR-539.

Author Contributions: Mireia Farrés-Cebrián performed the experiments and analysed the data regarding HPLC-UV optimization with the supervision of Raquel Seró. Mireia Farrés-Cebrián also peformed the application analysis and the chemometric study. Both Mireia Farrés-Cebrián and Raquel Seró worked under the close supervision of Javier Saurina and Oscar Núñez, who designed the experiments and wrote the paper.

Conflicts of Interest: The authors declare no conflict of interest.

\section{References}

1. Solomons, N.W. Evolutionary Aspects of Nutrition and Health: Diet, Exercise, Genetics and Chronic Disease, volume 84 of World Review of Nutrition and Dietetics edited by Artemis P. Simopoulos. Am. J. Clin. Nutr. 2000, 71, 854-855.

2. Simopoulos, A.P. The mediterranean diets: What is so special about the diet of Greece? The scientific evidence. J. Nutr. 2001, 131, 3065S-3073S. [PubMed] 
3. Stradling, C.; Hamid, M.; Fisher, K.; Taheri, S.; Thomas, G.N. A review of dietary influences on cardiovascular health: Part 1: The role of dietary nutrients. Cardiovasc. Hematol. Disord. Drug Targets 2013, 13, 208-230. [CrossRef] [PubMed]

4. Widmer, R.J.; Flammer, A.J.; Lerman, L.O.; Lerman, A. The Mediterranean diet, its components, and cardiovascular disease. Am. J. Med. 2015, 128, 229-238. [CrossRef] [PubMed]

5. Mediterranean diet. United Nation Educational, Scientific and Cultural Organization (UNESCO). Available online: http://www.unesco.org/culture/ich/en/RL/mediterranean-diet-00884 (accessed on 15 October 2016).

6. Helsing, E. Trends in fat consumption in Europe and their influence on the Mediterranean diet. Eur. J. Clin. Nutr. 1993, 47 (Suppl. 1), S4-S12. [PubMed]

7. European Union Commission. Council Regulation (EC) No. 1513/2001 of 23 July 2001 amending Regulations No. 136/66/EEC and (EC) No. 1638/98 as regards the extension of the period of validity of the aid scheme and the quality strategy for olive oil. Off. J. Eur. Communities 2001, L201, 4-7.

8. Rafehi, H.; Ververis, K.; Karagiannis, T.C. Mechanisms of Action of Phenolic Compounds in Olive. J. Diet. Suppl. 2012, 9, 96-109. [CrossRef] [PubMed]

9. Fedeli, E. Lipids of olives. Prog. Chem. Fats Other Lipids 1977, 15, 57-74. [CrossRef]

10. Rossell, J.B. Frying: Improving Quality; Woodhead Publishing Limited, CRC Press: Boca Raton, FL, USA, 2001.

11. Visioli, F.; Galli, C. Olive Oil Phenols and Their Potential Effects on Human Health. J. Agric. Food Chem. 1998, 46, 4292-4296. [CrossRef]

12. Bester, D.; Esterhuyse, A.J.; Truter, E.J.; van Rooyen, J. Cardiovascular effects of edible oils: A comparison between four popular edible oils. Nutr. Res. Rev. 2010, 23, 334-348. [CrossRef] [PubMed]

13. Waterman, E.; Lockwood, B. Active components and clinical applications of olive oil. Altern. Med. Rev. 2007, 12, 331-342. [PubMed]

14. Caponio, F.; Alloggio, V.; Gomes, T. Phenolic compounds of virgin olive oil: Influence of paste preparation techniques. Food Chem. 1999, 64, 203-209. [CrossRef]

15. Vekiari, S.A.; Koutsaftakis, A. The effect of different processing stages of olive fruit on the extracted olive oil polyphenol content. Grasas Aceites 2002, 53, 304-308. [CrossRef]

16. Motilva, M.J.; Tovar, M.J.; Romero, M.P.; Alegre, S.; Girona, J. Evolution of oil accumulation and polyphenol content in fruits of olive tree (Olea europaea L.) related to different irrigation strategies. Acta Hortic. 2002, 586, 345-348. [CrossRef]

17. Saurina, J.; Sentellas, S. Determination of Phenolic Compounds in Food Matrices: Applications to Characterization and Authentication. In Fast Liquid Chromatography-Mass Spectrometry Methods in Food and Environmental Analysis; Núñez, O., Gallart-Ayala, H., Martins, C.P.B., Lucci, P., Eds.; Imperial College Press: London, UK, 2015; pp. 517-547.

18. Carrasco-Pancorbo, A.; Cerretani, L.; Bendini, A.; Segura-Carretero, A.; Gallina-Toschi, T.; Fernandez-Gutierrez, A. Analytical determination of polyphenols in olive oils. J. Sep. Sci. 2005, 28, 837-858. [CrossRef] [PubMed]

19. Segura-Carretero, A.; Carrasco-Pancorbo, A.; Bendini, A.; Cerretani, L.; Fernandez-Gutierrez, A. Analytical determination of polyphenols in olive oil. Olives Olive Oil Health Dis. Prev. 2010, 509-523.

20. Gosetti, F.; Bolfi, B.; Manfredi, M.; Calabrese, G.; Marengo, E. Determination of eight polyphenols and pantothenic acid in extra-virgin olive oil samples by a simple, fast, high-throughput and sensitive ultrahigh performance liquid chromatography with tandem mass spectrometry method. J. Sep. Sci. 2015, 38, 3130-3136. [CrossRef] [PubMed]

21. Purcaro, G.; Codony, R.; Pizzale, L.; Mariani, C.; Conte, L. Evaluation of total hydroxytyrosol and tyrosol in extra virgin olive oils. Eur. J. Lipid Sci. Technol. 2014, 116, 805-811. [CrossRef]

22. Gouvinhas, I.; Machado, J.; Gomes, S.; Lopes, J.; Martins-Lopes, P.; Barros, A.I.R.N. Phenolic composition and antioxidant activity of monovarietal and commercial Portuguese olive oils. J. Am. Oil Chem. Soc. 2014, 91, 1197-1203. [CrossRef]

23. De la Torre-Robles, A.; Rivas, A.; Lorenzo-Tovar, M.L.; Monteagudo, C.; Mariscal-Arcas, M.; Olea-Serrano, F. Estimation of the intake of phenol compounds from virgin olive oil of a population from southern Spain. Food Addit. Contam. A 2014, 31, 1460-1469. [CrossRef] [PubMed]

24. Capriotti, A.L.; Cavaliere, C.; Crescenzi, C.; Foglia, P.; Nescatelli, R.; Samperi, R.; Lagana, A. Comparison of extraction methods for the identification and quantification of polyphenols in virgin olive oil by ultra-HPLC-QTOF mass spectrometry. Food Chem. 2014, 158, 392-400. [CrossRef] [PubMed] 
25. Longobardi, F.; Ventrella, A.; Casiello, G.; Sacco, D.; Tasioula-Margari, M.; Kiritsakis, A.K.; Kontominas, M.G. Characterisation of the geographical origin of Western Greek virgin olive oils based on instrumental and multivariate statistical analysis. Food Chem. 2012, 133, 169-175. [CrossRef]

26. Bayram, B.; Esatbeyoglu, T.; Schulze, N.; Ozcelik, B.; Frank, J.; Rimbach, G. Comprehensive Analysis of Polyphenols in 55 Extra Virgin Olive Oils by HPLC-ECD and Their Correlation with Antioxidant Activities. Plant Foods Hum. Nutr. 2012, 67, 326-336. [CrossRef] [PubMed]

27. Dais, P.; Boskou, P. Detection and quantification of phenolic compounds in olive oil, olives, and biological fluids. Olive Oil 2009, 55-107.

28. Garcia-Villalba, R.; Carrasco-Pancorbo, A.; Zurek, G.; Behrens, M.; Baessmann, C.; Segura-Carretero, A.; Fernandez-Gutierrez, A. Nano and rapid resolution liquid chromatography-electrospray ionization-time of flight mass spectrometry to identify and quantify phenolic compounds in olive oil. J. Sep. Sci. 2010, 33, 2069-2078. [CrossRef] [PubMed]

29. Bonoli, M.; Montanucci, M.; Toschi, T.G.; Lercker, G. Fast separation and determination of tyrosol, hydroxytyrosol and other phenolic compounds in extra-virgin olive oil by capillary zone electrophoresis with ultraviolet-diode array detection. J. Chromatogr. A 2003, 1011, 163-172. [CrossRef]

30. Bonoli, M.; Toschi, T.G.; Lercker, G. Analysis and quantification of phenolic compounds in vegetable extracts by high-performance capillary electrophoresis (HPCE). Prog. Nutr. 2005, 7, 31-35.

31. Beltran, G.; Jimenez, A.; Aguilera, M.P.; Uceda, M. HPLC analysis of the phenolic fraction of Arbequina virgin olive oil. Relations to the bitterness index K225 and oil stability. Grasas Aceites 2000, 51, 320-324. [CrossRef]

32. Marini, F.; D'Aloise, A.; Bucci, R.; Buiarelli, F.; Magri, A.L.; Magri, A.D. Fast analysis of 4 phenolic acids in olive oil by HPLC-DAD and chemometrics. Chemom. Intell. Lab. Syst. 2011, 106, 142-149. [CrossRef]

33. Lozano-Sanchez, J.; Segura-Carretero, A.; Menendez, J.A.; Oliveras-Ferraros, C.; Cerretani, L.; Gutierrez, A. Prediction of Extra Virgin Olive Oil Varieties through Their Phenolic Profile. Potential Cytotoxic Activity against Human Breast Cancer Cells. J. Agric. Food Chem. 2010, 58, 9942-9955. [CrossRef] [PubMed]

34. Alarcon Flores, M.I.; Romero-Gonzalez, R.; Garrido Frenich, A.; Martinez Vidal, J.L. Analysis of phenolic compounds in olive oil by solid-phase extraction and ultrahigh performance liquid chromatography-tandem mass spectrometry. Food Chem. 2012, 134, 2465-2472. [CrossRef] [PubMed]

35. Gutierrez-Rosales, F.; Rios, J.J.; Gomez-Rey, M.L. Main polyphenols in the bitter taste of virgin olive oil. Structural confirmation by on-line high-performance liquid chromatography electrospray ionization mass spectrometry. J. Agric. Food Chem. 2003, 51, 6021-6025. [CrossRef] [PubMed]

36. Garcia-Villalba, R.; Carrasco-Pancorbo, A.; Nevedomskaya, E.; Mayboroda, O.A.; Deelder, A.M.; Segura-Carretero, A.; Fernandez-Gutierrez, A. Exploratory analysis of human urine by LC-ESI-TOF MS after high intake of olive oil: Understanding the metabolism of polyphenols. Anal. Bioanal. Chem. 2010, 398, 463-475. [CrossRef] [PubMed]

37. Eigenvector Research Incorporated. Powerful Resources for Intelligent Data Analysis. Available online: http:/ / www.eigenvector.com/software/solo.htm (accessed on 15 October 2016).

38. Massart, D.L.; Vandeginste, B.G.M.; Buydens, L.M.C.; de Jong, S.; Lewi, P.J.; Smeyers-Verbeke, J. Handbook of Chemometrics and Qualimetrics; Elsevier: Amsterdam, The Netherlands, 1997.

39. Pérez-Rafols, C.; Saurina, J. Liquid chromatographic fingerprints and profiles of polyphenolic compounds applied to the chemometric characterization and classification of beers. Anal. Methods 2015, 7, 8733-8739. [CrossRef]

(C) 2016 by the authors; licensee MDPI, Basel, Switzerland. This article is an open access article distributed under the terms and conditions of the Creative Commons Attribution (CC-BY) license (http://creativecommons.org/licenses/by/4.0/). 